\title{
Gamma rays from annihilations at the galactic center in a physical dark matter distribution
}

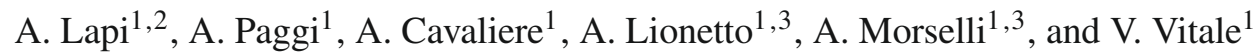 \\ 1 Dip. Fisica, Univ. “Tor Vergata”, via Ricerca Scientifica 1, 00133 Roma, Italy \\ e-mail: lapi@roma2.infn.it \\ 2 SISSA/ISAS, via Beirut 2-4, 34151 Trieste, Italy \\ 3 INFN-Sezione di Roma2, via Ricerca Scientifica 1, 00133 Roma, Italy
}

Received 25 September 2009 / Accepted 28 November 2009

\section{ABSTRACT}

\begin{abstract}
We discuss the $\gamma$-ray signal to be expected from dark matter (DM) annihilations at the Galactic center. We describe the DM distribution in the Galactic halo, based on the Jeans equation for self-gravitating, anisotropic equilibria. In solving the Jeans equation, we adopted the specific correlation between the density $\rho(r)$ and the velocity dispersion $\sigma_{r}^{2}(r)$ expressed by the powerlaw behavior of the DM "entropy" $K \equiv \sigma_{r}^{2} / \rho^{2 / 3} \propto r^{\alpha}$ with $\alpha \approx 1.25-1$.3. Indicated (among others) by several recent $N$-body simulations, this correlation is privileged by the form of the radial pressure term in the Jeans equation, and it yields a main-body profile consistent with the classic self-similar development of DM halos. In addition, we required the Jeans solutions to satisfy regular boundary conditions both at the center (finite pressure, round gravitational potential) and on the outskirts (finite overall mass). With these building blocks, we derived physical solutions, dubbed " $\alpha$-profiles". We find the one with $\alpha=1.25$, suitable for the Galaxy halo, to be intrinsically flatter at the center than the empirical NFW formula, yet steeper than the empirical Einasto profile. On scales of $10^{-1}$ deg it yields annihilation fluxes lower by a factor 5 than the former, yet higher by a factor 10 than the latter. Such fluxes will eventually fall within the reach of the Fermi satellite. We show the effectiveness of the $\alpha$-profile in relieving the astrophysical uncertainties related to the macroscopic DM distribution, and discuss its expected performance as a tool instrumental in interpreting the upcoming $\gamma$-ray data in terms of DM annihilation.
\end{abstract}

Key words. cosmology: dark matter - galaxies: evolution - Galaxy: halo - methods: analytical

\section{Introduction}

Several astrophysical and cosmological probes (for a review see Bertone et al. 2005) have firmly established that baryons - which stars, planets, and (known) living creatures are made of - constitute only some $15 \%$ of the total matter content in the Universe (adding to the dominant dark-energy component). The rest is in the form of "cold dark matter" (DM), i.e., massive particles that were nonrelativistic at decoupling, do not emit/absorb radiations, and basically do not interact with themselves and with the baryons except via long-range gravitational forces.

However, no "direct" detection of the DM has been made so far, other than Bernabei et al. (2008). Thus the microscopic nature of the DM largely remains a mystery, where several clues suggest as a promising candidate or component the lightest supersymmetric particle, the "neutralino" (for a review see Bertone 2009). Given that the latter's mass, depending on the specific supersymmetric model, ranges from several $\mathrm{GeVs}$ to tens of $\mathrm{TeVs}$, its laboratory production requires an accelerator at least as powerful as the newly-born Large Hadron Collider (see Baer \& Tata 2009). The discovery of supersymmetry and specifically of the neutralino is one of the main aims for the current experiments in high-energy physics.

Meanwhile, evidence of the DM can be looked for "indirectly" in the sky. In fact, the basic aims of the recently launched Fermi satellite include the search for $\gamma$-ray signals due to the annihilation of DM particles at the Galactic center (GC) and in nearby galaxies (see discussion in Sect. 4). The former provides a favorable target because it is closest to us, with the DM density expected to increase in moving toward the inner regions of a galaxy. However, the GC is also a crowded region, and it remains a challenging task to separate the DM signal from the contributions of other astrophysical sources and backgrounds whose energy spectrum and angular distribution are poorly known.

In principle, if one can predict the strength and angular distribution of the annihilation signal itself, then the $\gamma$-ray observations would elicit, or put "indirect" constraints on, the (combined) microscopic properties of the DM particles like mass, annihilation cross section, and channels. This approach has been pursued extensively (e.g., Bergström et al. 1998; Fornengo et al. 2004; Strigari 2007; Bertone et al. 2009; Serpico \& Hooper 2009) but still suffers from large uncertainties (see Cesarini et al. 2004), mainly related to the poor knowledge of the macroscopic DM distribution $\rho(r)$ throughout the Galaxy. Since the annihilation rate scales like $\rho^{2}(r)$, such uncertainties are maximized near the center right where detection is favored. Similar if milder uncertainties affect the source function of the electrons originating in DM annihilations by production or cascading; these diffuse outwards and interact with the Galactic magnetic field and with the interstellar light to produce synchrotron emission observed in the radio band (see Bertone et al. 2009), and inverse Compton radiation observable in $\gamma$ rays (see Papucci \& Strumia 2009).

Traditionally, the density profile of an equilibrium DM structure, or "halo", is rendered in terms of different empirical formulas that fit the results of $N$-body simulations and, to some extent, the stellar observations. Perhaps the most popular one is the Navarro, Frenk \& White (hereafter NFW; see Navarro et al. 1997) profile, which has an asymptotic inner slope $\rho(r) \propto r^{-1}$, 
goes over to a powerlaw behavior $\rho(r) \propto r^{-2}$ in the halo's middle, and declines as $\rho(r) \propto r^{-3}$ in the outer regions. Despite its widespread use in the literature, clearly this expression cannot account for the actual DM distribution in the inner regions of a galaxy halo where it would imply a centrally angled gravitational potential well and an infinite pressure, nor in the halo outskirts where it would yield a diverging overall mass.

Other empirical density profiles have been proposed, but they suffer of similarly unphysical features; e.g., the Moore profile (see Diemand et al. 2005) goes like $\rho(r) \propto r^{-1.2}$ and implies a gravitational force diverging towards the center, while the Einasto profile (see Graham et al. 2006) behaves like $\rho(r) \propto$ $\mathrm{e}^{-a r^{b}}$, so it yields a vanishing pressure there. We stress that the differences in the predicted annihilation signals under these DM distributions turn out to be quite considerable; e.g., the ratio of the NFW to the Einasto squared density averaged over 1 degree (about $150 \mathrm{pc}$ ) comes to a factor 10 when normalized at the Sun's location (see also Sect. 4).

Here our stand is that the macroscopic uncertainties yielding such differences can and ought to be relieved. To this purpose, in Sect. 2 we present the physical density distributions that we $\operatorname{dub} \alpha$-profiles. These are solutions of the Jeans equation that satisfy regular inner and outer boundary conditions. In Sect. 3 we use the $\alpha$-profile suitable for the Galaxy halo as the macroscopic benchmark for evaluating the DM annihilation signal expected from the GC. We base the microscopic sector on a standard model for the mass, cross section, and annihilation channel of the DM particles, because the extension to more complex microphysics is straightforward. Finally, our findings are summarized and discussed in Sect. 4.

Throughout this work we adopt a standard, flat cosmology (see Dunkley et al. 2009) with normalized matter density $\Omega_{M}=$ 0.27 , and Hubble constant $H_{0}=72 \mathrm{~km} \mathrm{~s}^{-1} \mathrm{Mpc}^{-1}$.

\section{Development and structure of DM halos}

Galaxies are widely held to form under the drive of the gravitational instability that acts on initial perturbations modulating the cosmic density of the dominant cold DM component. At first the instability is kept in check by the cosmic expansion, but when the local gravity prevails, collapse sets in, to form a DM halo in equilibrium under self-gravity. The amplitude of more massive perturbations is smaller, so the formation is progressive in time and hierarchical in mass, with the largest structures typically forming later (see Peebles et al. 1983, for a review).

\subsection{Two-stage evolution}

Such a formation history has been resolved to a considerable detail by many $N$-body simulations (e.g., White 1986; Springel et al. 2006); recently, a novel viewpoint emerged. First, the halo growth has been recognized (see Zhao et al. 2003; Wechsler et al. 2006; Hoffman et al. 2007; Diemand et al. 2007) to comprise two stages: an early fast collapse including a few violent major mergers, that builds up the halo main "body" with the structure set by dynamical relaxation; and a later, quasi-equilibrium stage when the body is nearly unaffected, while the outskirts develop from the inside-out by minor mergers and smooth accretion (see Salvador-Sole et al. 2007). The transition is provided by the time when a DM gravitational potential attains its maximal depth; i.e., the radial peak of the circular velocity $v_{\mathrm{c}}^{2} \equiv G M / R$ attains its maximal height, along a given growth history (see Li et al. 2007).
Second, generic features of the ensuing equilibrium structures have been sought (see Hansen 2004; Dehnen \& McLaughlin 2005; Schmidt et al. 2008) among powerlaw correlations of the form $\sigma_{D}^{2 \epsilon / 3} / \rho^{2 / 3} \propto r^{\alpha}$; this involves the density $\rho(r)$ and the velocity dispersion $\sigma_{D}^{2} \equiv \sigma_{r}^{2}(1+D \beta)$, with anisotropy inserted via the standard Binney (1978) parameter $\beta \equiv 1-\sigma_{\theta}^{2} / \sigma_{r}^{2}$ and modulated by the index $D$ (see Hansen 2007). It is matter of debate which of these correlations best apply, see Schmidt et al. (2008) and Navarro et al. (2010); the former authors, in particular, find that the structure of different simulated halos may be described by different values of $D$, with linearly related values of $\epsilon$ and $\alpha$ (see their Eqs. (4) and (5)).

Here we focus on the specific correlation

$K \equiv \frac{\sigma_{r}^{2}}{\rho^{2 / 3}} \propto r^{\alpha}$

that solely involves the squared radial dispersion $\sigma_{r}^{2}$, corresponding to $D=0$ and $\epsilon=3$. This is because $K$ has not only the striking form of a DM "entropy" (or rather adiabat), but also the related operational advantage of providing a direct expression of the radial pressure term $\rho \sigma_{r}^{2}=K \rho^{5 / 3} \propto r^{\alpha} \rho^{5 / 3}$ appearing in the equilibrium Jeans equation, wherein any anisotropy is already accounted for by a separate term (see Eq. (2) below). It is also widely accepted that the correlation $K \propto r^{\alpha}$ with $\alpha \approx 1.25-1.3$ provides a simple but still effective fit of many simulations (see Taylor \& Navarro 2001; Rasia et al. 2004; Hoffman et al. 2007; Diemand et al. 2007; Schmidt et al. 2008; Ascasibar \& Gottlöber 2008; Navarro et al. 2010; Vass et al. 2008, and many others). In the lower $\alpha$ range, Eq. (1) has the added bonus of preserving the classic self-similar slope in the halo body (see Eq. (3) below).

To independently probe the matter, Lapi \& Cavaliere (2009a) performed a semianalytical study of the two-stage halo development, and derived (consistent with the simulations) that $\alpha$ is set at the transition time via scale-free stratification of the particle orbits throughout the halo body, and thereafter remains closely constant and uniform at a value within the narrow range 1.251.3. Moreover, they find that on average the values of $\alpha$ weakly depend on the mass of the halo, such that $\alpha \approx 1.3$ applies to galaxy clusters, while $\alpha \approx 1.25$ applies to Milky Way sized galaxies.

\subsection{The DM $\alpha$-profiles}

The halo physical profiles may be derived from the radial Jeans equation, with the radial pressure $\rho \sigma_{r}^{2} \propto r^{\alpha} \rho^{5 / 3}$ and anisotropies described by the standard Binney (1978) parameter $\beta$. Thus the Jeans equation simply writes as

$\gamma=\frac{3}{5}\left(\alpha+\frac{v_{c}^{2}}{\sigma_{r}^{2}}\right)+\frac{6}{5} \beta$

in terms of the logarithmic density slope $\gamma \equiv-\mathrm{d} \log \rho / \mathrm{d} \log r$. As first shown by Austin et al. (2005) and Dehnen \& McLaughlin (2005), Jeans supplemented with the mass definition $M(<r) \equiv$ $4 \pi \int_{0}^{r} \mathrm{~d} r^{\prime} r^{\prime 2} \rho\left(r^{\prime}\right)$ entering $v_{\mathrm{c}}^{2} \equiv G M(<r) / r$ provides an integrodifferential equation for $\rho(r)$, which by double differentiation reduces to a handy 2nd-order differential equation for $\gamma$.

To set the context for the Milky Way DM distribution, we recall that the space of solutions for Eq. (2) spans the range $\alpha \leq$ 1.296: the one for the upper bound and the behaviors of others ones have been analytically investigated by Austin et al. (2005) and Dehnen \& McLaughlin (2005). In Lapi \& Cavaliere (2009a), we explicitly derived the Jeans solutions with $\beta=0$ (meaning 

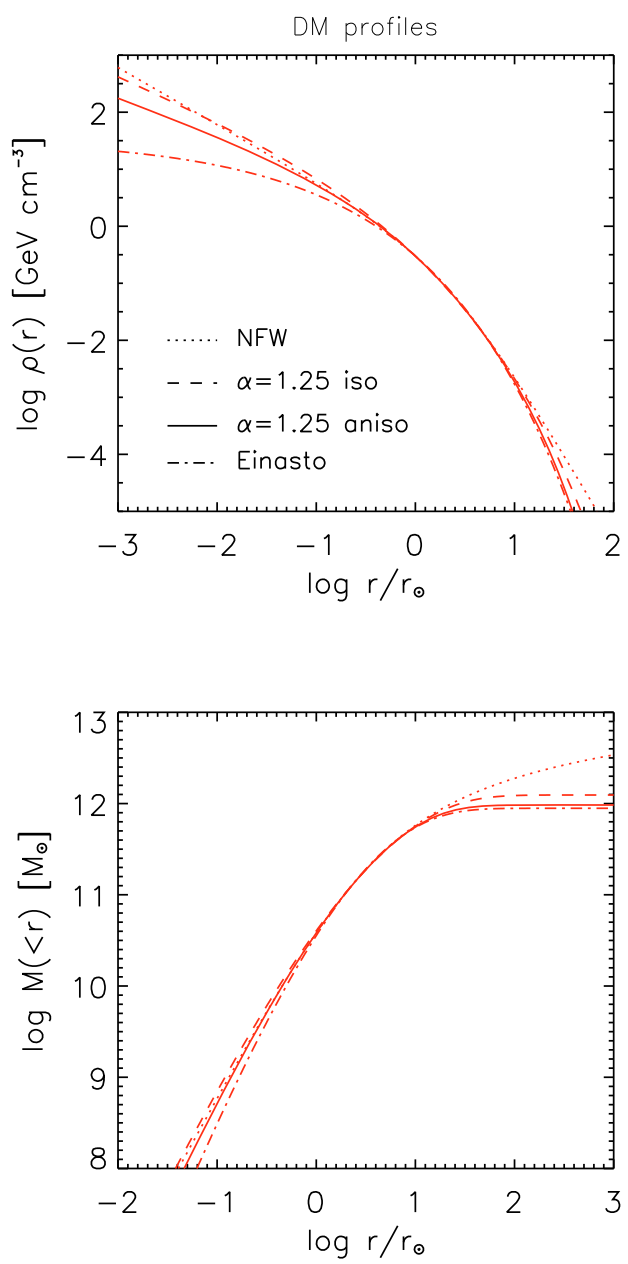

Fig. 1. Density and mass profiles in the Milky Way. The dashed and solid lines illustrate the $\alpha$-profiles with $\alpha=1.25$ in the isotropic and the anisotropic cases, with $\gamma_{a}=0.75$ and 0.63 , respectively. The dotted line represents the NFW formula, and the dot-dashed line refers to the Einasto profile. All profiles are normalized to the local density $0.3 \mathrm{GeV} \mathrm{cm}{ }^{-3}$ at the Sun's location $r_{\odot} \approx 8.5 \mathrm{kpc}$ within the Galaxy; we have adopted $r_{-2}=20 \mathrm{kpc}$ and $c=10$, see Sect. 2 .

isotropy) for the full range $\alpha \approx 1.25-1 . \overline{296}$ subjected to regular boundary conditions both at the center and in the outskirts, i.e., a round minimum of the potential with a finite pressure (or energy density) and a finite (hence definite) overall mass, respectively. These we dubbed " $\alpha$-profiles".

The corresponding density runs steepen monotonically outwards and are summarized by the pivotal slopes

$\gamma_{a} \equiv \frac{3}{5} \alpha, \quad \gamma_{0} \equiv 6-3 \alpha, \quad \gamma_{b} \equiv \frac{3}{2}(1+\alpha)$.

These start from the central $(r \rightarrow 0)$ value $\gamma_{a} \approx 0.75-078$, steepen in the halo main body to $\gamma_{0} \approx 2.25-2.1$ (the former being the slope from the classic self-similar collapse), and steepen further into the outskirts to typical values $\gamma_{b} \approx 3.38-3.44$ before a final cutoff. Thus the inner slope is considerably flatter and the outer slope steeper compared to the empirical NFW formula (see Navarro et al. 1997). In comparison to the Einasto profile, the main difference occurs in the inner regions where the $\alpha$-profile is moderately steep rather than flat (see Eq. (3) and Fig. 1).

For a density profile, a relevant parameter is the concentration $c \equiv R_{v} / r_{-2}$, defined in terms of the virial radius $R_{v}$ and of the radius $r_{-2}$ where $\gamma=2$. In the context of $\alpha$-profiles $c$ may be viewed as a measure either of central condensation (small $r_{-2}$ ) or of outskirts' extension (large $R_{v}$ ). The concentration constitutes an indicator of the halo age; in fact, numerical experiments (see Bullock et al. 2001; Zhao et al. 2003; Wechsler et al. 2006; Diemand et al. 2007) show that $c(z) \approx 3.5$ holds at the end of the fast collapse stage, to grow as $c(z) \approx 3.5\left(1+z_{\mathrm{t}}\right) /(1+z)$ during the slow accretion stage after the transition at $z_{\mathrm{t}}$. Current values $c \approx 3.5\left(1+z_{\mathrm{t}}\right) \approx 10$ apply for a galaxy like the Milky Way that had its transition at $z_{\mathrm{t}} \lesssim 2$.

The density and mass distribution in the Milky Way are illustrated in Fig. 1 for the isotropic $\alpha$-profiles with $\alpha=1.25$, for the NFW formula, and for the Einasto profile. All densities have been normalized to the local density $0.3 \mathrm{GeV} \mathrm{cm}^{-3}$ at the Sun's location $r_{\odot} \approx 8.5 \mathrm{kpc}$ within the Galaxy. We further adopt $r_{-2}=20 \mathrm{kpc}$ (consistent with $c=10$ ). Note in Fig. 1 that the Einasto and NFW profiles differ substantially at the center as for the density and in the outskirts as for the mass, while the $\alpha$-profile strikes an intermediate course between the two.

\subsection{Anisotropy}

It is clear from Eq. (2) that anisotropy will steepen the density run for positive $\beta$ meaning radial velocity dominance, as expected in the outskirts from infalling cold matter. On the other hand, tangential components (corresponding to $\beta \lesssim 0$ ) must develop toward the center, as expected from the increasing strength of angular momentum effects. This view is supported by numerical simulations (see Austin et al. 2005; Hansen \& Moore 2006; Dehnen \& McLaughlin 2005), which in detail suggest the effective linear approximation

$\beta(r) \approx \beta(0)+\beta^{\prime}\left[\gamma(r)-\gamma_{a}\right]$

to hold with $\beta(0) \geq-0.1$ and $\beta^{\prime} \approx 0.2$, limited to $\beta(r)<0.5$.

In Lapi \& Cavaliere (2009b) we extended the $\alpha$-profiles to such anisotropic conditions in the full range $\alpha \approx 1.25-1.3$, inspired by the analysis by Dehnen \& McLaughlin (2005) for the specific case $\alpha \approx 1.3$. We find the corresponding $\rho(r)$ to be somewhat flattened at the center by a weakly negative $\beta(0)$ and further steepened into the outskirts where $\beta(r)$ grows substantially positive. Specifically, the following simple rules turn out to apply: the slope $\beta^{\prime}$ in Eq. (4) drops out from the derivatives of the Jeans equation (see Dehnen \& McLaughlin 2005); the upper bound to $\alpha$ now reads $35 / 27-4 \beta(0) / 27$; moreover, $\gamma_{a}$ is modified into $3 \alpha / 5+6 \beta(0) / 5$, while $\gamma_{0}$ and $\gamma_{b}$ retain their form.

The anisotropic $\alpha$-profiles for the Milky Way are shown in Fig. 1. We note, in particular, that even a limited central anisotropy (e.g., $\beta[0] \approx-0.1$ ) causes an appreciable flattening of the inner density slope, bringing it down to $\gamma_{a} \approx 0.63$ for $\alpha=1.25$. Plainly, this result produces an even more considerable flattening in the slope of the squared density, the relevant quantity in our context of DM annihilations.

\subsection{A guide to profile computations}

Finally, in the Appendix we provide user-friendly analytic fits for the density runs of the $\alpha$-profiles in terms of standard deprojected Sérsic formulas, but with parameters directly derived from the Jeans equation. We stress that these physical $\alpha$-profiles with their analytic fits are relevant to, and recently tested in several contexts, including the interpretation of gravitational lensing observations (see Lapi \& Cavaliere 2009b), the physics of the hot diffuse baryons constituting the intra-cluster plasma (see Cavaliere et al. 2009), and galaxy kinematics (Lapi \& Cavaliere, in preparation). In the following we focus on the specific 
$\alpha$-profile with $\alpha=1.25$ suitable for the Milky Way halo (see Sect. 2.1) to predict the DM annihilation signal from the GC.

\section{3. $\gamma$-ray signal from DM annihilation at the GC}

The $\gamma$-ray flux per solid angle due to DM annihilation along a direction at an angle $\psi$ relative to the line-of-sight toward the GC may be written (under the commonly assumed spherical symmetry) as

$\frac{\mathrm{d} \Phi_{\gamma}}{\mathrm{d} \Omega}=3.74 \times 10^{-6} N_{\gamma}\left(\frac{\langle\Sigma v\rangle}{10^{-26} \mathrm{~cm}^{3} \mathrm{~s}^{-1}}\right) \quad\left(\frac{m_{\mathrm{DM}}}{50 \mathrm{GeV}}\right)^{-2} J(\psi)$

in units of $\mathrm{m}^{-2} \mathrm{~s}^{-1} \mathrm{sr}^{-1}$. This is naturally factorized into a $m i$ croscopic and an astrophysical term (e.g., Bergström 2009, and references therein). The former involves the mass of the DM particle $m_{\mathrm{DM}}$, the number of photons $N_{\gamma}$ created per annihilation, and the angle-velocity averaged annihilation rate $\langle\Sigma v\rangle$ in terms of the particles' cross section $\Sigma$ and velocity $v$.

For the sake of definiteness we begin by considering a neutralino DM particle with mass $m_{\mathrm{DM}} \approx 50 \mathrm{GeV}$, annihilating through the $b \bar{b}$ channel (with $100 \%$ branching ratio). We use the benchmark value for the annihilation rate $\langle\Sigma v\rangle \approx 3 \times$ $10^{-26} \mathrm{~cm}^{3} \mathrm{~s}^{-1}$, corresponding to a thermal relic with a density close to the cosmological DM abundance

$\Omega_{\mathrm{DM}} h^{2} \approx \frac{3 \times 10^{-27} \mathrm{~cm}^{3} \mathrm{~s}^{-1}}{\langle\Sigma v\rangle} \approx 0.1$

as measured by WMAP (see Dunkley et al. 2009). To compute $N_{\gamma}=\int \mathrm{d} E \mathrm{~d} N_{\gamma} / \mathrm{d} E_{\gamma}$, we adopt a photon annihilation spectrum with shape

$\frac{\mathrm{d} N_{\gamma}}{\mathrm{d} x}=\eta x^{a} \mathrm{e}^{b+c x+d x^{2}+e x^{3}}$,

obtained from extrapolating the results by Fornengo et al. (2004) down to energies $E_{\gamma} \approx 200 \mathrm{MeV}$. Here $x \equiv E_{\gamma} / m_{\mathrm{DM}}$ is the energy normalized to the DM mass, while $\eta=1, a=-1.5$, $b=0.579, c=-17.6080, d=23.862, e=-25.181$ are fitting parameters for the adopted microscopic DM model (see above).

The astrophysical term of Eq. (5) is given by the integral of the (squared) DM density projected along the line-of-sight

$J(\psi)=\int \frac{\mathrm{d} \ell}{r_{\odot}} \frac{\rho^{2}(r)}{\rho^{2}\left(r_{\odot}\right)}$,

normalized to $\rho\left(r_{\odot}\right) \approx 0.3 \mathrm{GeV} \mathrm{cm} \mathrm{cm}^{-3}$, the local density at the Sun's location $r_{\odot} \approx 8.5 \mathrm{kpc}$. A non-trivial angular dependence results from the peripheral position of the Sun within the Milky Way halo, and only involves the angle $\psi$ between the observed direction of the sky and the GC. In terms of Galactic latitude $b$ and longitude $l$ with $\cos \psi=\cos b \cos l$, the radial variable can be expressed as $r=\left(r_{\odot}^{2}+\ell^{2}-2 r_{\odot} \ell \cos \psi\right)^{1 / 2}$ on using the distance $\ell$ along the line-of-sight. Finally, when observing a region at an angular resolution $\Delta \Omega$, one has to consider the average value of $J$, namely,

$\bar{J}=\int_{\Delta \Omega} \mathrm{d} \Omega J(\psi)$,

with $\mathrm{d} \Omega=\cos b \mathrm{~d} b \mathrm{~d} l$.

We computed and report in Table 1 the values of $\bar{J}$ at angular resolutions $\Delta \Omega=10^{-3} \mathrm{sr}$ and $10^{-5} \mathrm{sr}$ for the $\alpha$-profile with $\alpha=1.25$ in the isotropic and anisotropic cases, for the NFW
Table 1. Values of the astrophysical factor $\bar{J}$.

\begin{tabular}{ccc}
\hline \hline DM profile & $\Delta \Omega=10^{-3}$ & $\Delta \Omega=10^{-5}$ \\
\hline NFW & $1.2 \times 10^{3}$ & $1.05 \times 10^{4}$ \\
$\alpha$ iso & $1.3 \times 10^{3}$ & $6.5 \times 10^{3}$ \\
$\alpha$ aniso & $5.1 \times 10^{2}$ & $1.6 \times 10^{3}$ \\
Einasto & $1.1 \times 10^{2}$ & $1.3 \times 10^{2}$ \\
\hline
\end{tabular}

Table 2. Values of the $\gamma$-ray flux (in $\mathrm{m}^{-2} \mathrm{~s}^{-1}$ ) for $E_{\gamma} \geq 200 \mathrm{MeV}$.

\begin{tabular}{ccc}
\hline \hline DM profile & $\Delta \Omega=10^{-3}$ & $\Delta \Omega=10^{-5}$ \\
\hline NFW & $4.7 \times 10^{-4}$ & $4.0 \times 10^{-5}$ \\
$\alpha$ iso & $5.2 \times 10^{-4}$ & $2.5 \times 10^{-5}$ \\
$\alpha$ aniso & $2.0 \times 10^{-4}$ & $6.0 \times 10^{-6}$ \\
Einasto & $4.1 \times 10^{-5}$ & $5.0 \times 10^{-7}$ \\
\hline
\end{tabular}
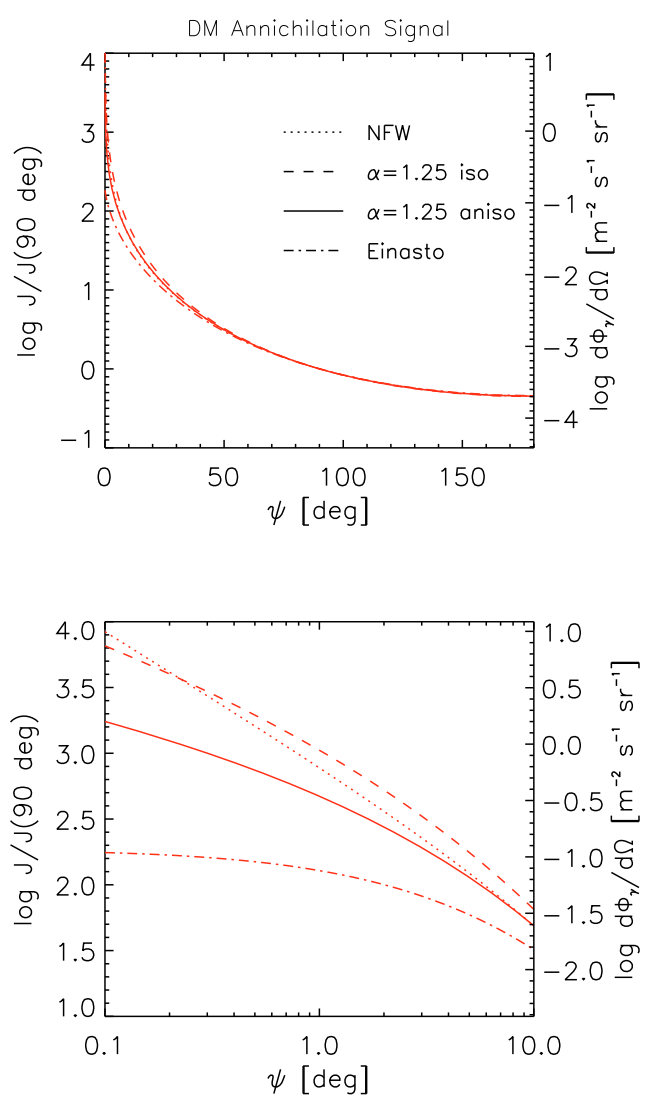

Fig. 2. The astrophysical factor $J(\psi)$ normalized to the value at $\psi=$ 90 deg (left axis), and the corresponding annihilation flux per unit solid angle for $E_{\gamma} \geq 200 \mathrm{MeV}$ (right axis; see Sect. 3 for details). The bottom panel zooms on the inner angular scales.

formula, and for the Einasto profile. In Table 2 we list the corresponding values of the $\gamma$-ray flux for energies $E_{\gamma} \geq 200 \mathrm{MeV}$. These outcomes are illustrated in Fig. 2. It is seen that, relative to the NFW distribution, the fluxes predicted from the isotropic and anisotropic $\alpha$-profile are lower by factors from a few to several. Such fluxes are still within the reach of the Fermi satellite; in fact, on the basis of the simulations performed by Baltz et al. (2008), Striani (2009), and Vitale et al. (2009a), we expect the annihilation signal to be probed at a $3-\sigma$ confidence level over a few years.

The above values may be compared with the current upper bound to the integrated flux of $2.43_{-0.02}^{+0.02} \times 10^{-3} \mathrm{~m}^{-2} \mathrm{~s}^{-1}$ based on Fermi measurements at $E_{\gamma} \geq 200 \mathrm{MeV}$ during a 8-month 
observation of the GC over a solid angle $\Delta \Omega \approx 10^{-3} \operatorname{sr}$ (see Abdo et al. 2009; Atwood et al. 2009; Vitale et al. 2009b); this bound will decrease as $1 / \sqrt{t}$ with the observation time $t$. However, the flux observed currently includes contributions from diffuse or not-yet-resolved Galactic sources, which are being progressively removed (see Goodenough \& Hooper 2009; Striani 2009; Vitale et al. 2009b). Next stages of such a process will take longer observations aimed at determining the spectrum of individual resolved sources and a careful likelihood analysis of the backgrounds (see discussion by Cesarini et al. 2004).

\section{Discussion and conclusions}

We have presented our $\alpha$-profile with $\alpha=1.25$ for the equilibrium density and mass distributions in a galactic DM halo, and specifically in the Milky Way. We have shown that this profile constitutes the robust solution of the equilibrium Jeans equation with physical inner and outer boundary conditions, i.e., finite pressure and round potential minimum at the center and finite overall mass. The corresponding density profile $\rho(r)$ is intrinsically flatter at the center and intrinsically steeper in the outskirts, relative to the empirical NFW formula. These features are still sharpened in halos with anisotropic random velocities. We also provided the reader with a precise and user-friendly analytic fit to the $\alpha$-profile (see Appendix for details).

Then we focused on the role of this $\alpha$-profile as a benchmark for computing the DM annihilation signal expected from the GC. In fact, we computed the "astrophysical factor" $J(\psi)$ (angular distribution, independent of microphysics) entering the expression of the annihilation flux. As a definite example, we also computed the $\gamma$-ray flux on adopting a simple, fiducial microscopic model. This we find consistent with current Fermi observations, which may include contributions from still unresolved point sources.

Given the physical $\alpha$-profile and the corresponding factor $J(\psi)$, the extension to more complex microscopic scenarios like mSUGRA (started by Chamseddine et al. 1982; Barbieri et al. 1982; Ohta et al. 1983; Hall et al. 1983) will be easily made in terms of annihilations channels, cross sections and particle masses. In this context our $\alpha$-profile relieves astrophysical uncertainties related to the macroscopic DM distribution. We stress that constraints on particle cross sections and masses inferred from radio and $\gamma$-ray observations of the GC have been to now more sensitive to the assumed DM distribution than to specific annihilation channels (different from leptonic $\tau \bar{\tau}$ ), see Figs. 3 and 4 in Bertone et al. (2009). In fact, the latter show that a DM distribution with an inner slope like our $\alpha$-profile is required to allow cross sections $\langle\Sigma v\rangle \gtrsim 10^{-26} \mathrm{~cm}^{3} \mathrm{~s}^{-1}$ with masses $m_{\mathrm{DM}} \lesssim 500 \mathrm{GeV}$ for the non-leptonic channels, which are widely considered on grounds of theoretical microphysics.

Concerning small scales $r \sim$ a few tens of pcs around the GC, we touch upon a number of possible deviations of the very inner DM density distribution from our benchmark $\alpha$-profile (Fig. 1, top panel). For example, the process of galaxy formation could lead either to flattening or to some steepening of the inner DM distribution. The former may occur either owing to transfer of energy and/or angular momentum from the baryons to the DM (see El-Zant et al. 2001; Tonini et al. 2006) or owing to quick mass removal following the energy feedback from stars or active galactic nuclei (see discussion by Lauer et al. 2007; Kormendy et al. 2009). On the other hand, steepening might be induced by the 'adiabatic' contraction of the baryons into the disk (see Blumenthal et al. 1986; Mo et al. 1998); but even in extreme cases (see discussion by Abadi et al. 2009) such a contraction would yield an inner DM density profile $\rho(r) \propto r^{-3 /\left(4-\gamma_{a}\right)}$, still flatter than 1 though somewhat steeper than the original $\gamma_{a} \approx$ $0.63-0.75$. Finally, at the very center of the Galaxy any accretion of DM (e.g., Gondolo et al. 1999; Bertone et al. 2002) onto the nuclear supermassive black hole might enhance the DM distribution on tiny scales $r<10^{-1} \mathrm{pc}$.

Summing up, we stress that all such alterations of the inner slope would occur on scales smaller than some 10 pcs. Although significant at levels of a few percent to account for the central stellar light ${ }^{1}$, their import is far smaller where the annihilation signal is concerned, and on the average over $10^{-1} \mathrm{deg}$ the flux is altered by less than $0.1 \%$. In fact, these corrections are currently at or below the resolution limit and the prospective sensitivity of Fermi.

Other possible targets include the dwarf spheroidal galaxies in the Local Group. On the one hand, these constitute cleaner environments than the GC owing to the dearth of stellar sources; on the other, their distance, if modest on intergalactic scales, already makes detecting and resolving the related annihilation signal a real challenge for Fermi (e.g., Pieri et al. 2009). In addition, the shallow gravitational potential wells of these systems make them particularly prone to energy feedback events (see above), which may flatten the inner DM distribution to flat slopes $\gamma_{a}<0.63$ (consistent with kinematical observations), with the effect of further lowering the annihilation signals. Upper limits more stringent than the current value $\langle\Sigma v\rangle<10^{-25} \mathrm{~cm}^{3} \mathrm{~s}^{-1}$ at a mass $m_{\mathrm{DM}} \approx 50 \mathrm{GeV}$ will require delicate stacking over an ensemble of dwarfs.

Concerning particle cross section and masses, we recall that the PAMELA satellite recently observed an excess of the positron fraction $\mathrm{e}^{+} /\left(\mathrm{e}^{+}+\mathrm{e}^{-}\right)$in the cosmic ray $\mathrm{e}^{ \pm}$spectra relative to the expected astrophysical background above $10 \mathrm{GeV}$ (see Adriani et al. 2009). This excess can simply be explained in terms of a single or a few sources like pulsars, which are expected to produce a powerlaw spectrum of $\mathrm{e}^{ \pm}$pairs with a cutoff at several TeVs (see Bertone 2009). On the other hand, the signal may also be interpreted in terms of DM annihilations occurring throughout the Galactic halo (e.g., Bertone et al. 2009).

If this is to be the case, however, the flux measured by PAMELA mandates for very large effective annihilation cross sections $\langle\Sigma v\rangle \sim 10^{-23} \mathrm{~cm}^{3} \mathrm{~s}^{-1}$, well above the natural value suggested by the cosmological DM abundance (see Sect. 3). From a microphysical point of view, this is still conceivable in scenarios with Sommerfeld enhancements (see discussion by Arkani-Hamed et al. 2009). The cross section may be enhanced by a factor $\sim 10^{2}$ for velocities $v / c \sim 10^{-3}$. On the other hand, such a large Sommerfeld effects would also yield a strong $\gamma$-ray annihilation signal towards the GC; for this, little room is allowed on the basis of the current upper limit provided by Fermi (see Sect. 3), unless the DM particle mass substantially exceeds $50 \mathrm{GeV}$.

Another possibility is to invoke a large boost factor of the effective cross section due to clumpiness in the Galactic halo, i.e., a crowd of dense subhalos; however, state-of-the-art numerical simulations suggest such boosts are not realistic in the Galaxy, even less at the GC (see Springel et al. 2008; and discussion by Lattanzi \& Silk 2009).

To sum up, we have discussed why the $\alpha$-profile with $\alpha=$ 1.25 (see Fig. 1) constitutes a reliable DM distribution in the Galaxy. We argued that it will provide a benchmark to gauge the

\footnotetext{
1 A flat slope is known to describe the very central light distribution in luminous ellipticals, which relates to complex small-scale dynamics (see Lauer et al. 2007; Kormendy et al. 2009).
} 
Table A.1. Values of the fitting parameters of Eq. (A.1) in the isotropic case, where $\alpha=1.25$ applies for the Galactic halo.

\begin{tabular}{cccc}
\hline \hline$\alpha$ & 1.25 & 1.26 & 1.27 \\
\hline$\tau$ & 0.750 & 0.756 & 0.762 \\
$\eta$ & 0.319 & 0.298 & 0.277 \\
\hline
\end{tabular}

Table A.2. Values of the fitting parameters of Eq. (A.1) in the anisotropic case, where $\alpha=1.25$ applies for the Galactic halo.

\begin{tabular}{cccc}
\hline \hline$\alpha$ & 1.25 & 1.26 & 1.27 \\
\hline$\tau$ & 0.630 & 0.636 & 0.642 \\
$\eta$ & 0.364 & 0.342 & 0.319 \\
\hline
\end{tabular}

$\gamma$ rays from the GC to be detected with Fermi in terms of DM annihilation (see Fig. 2). Such an $\alpha$-profile will be instrumental in deriving reliable information concerning the microscopic nature of the DM particles.

Acknowledgements. This work was supported by Agenzia Spaziale Italiana (ASI), Istituto Nazionale di Astrofisica (INAF), and Istituto Nazionale di Fisica Nucleare (INFN). We acknowledge our referee S. H. Hansen for keen and helpful comments. We are indebted to L. Ciotti, A. Cirelli, P. Salucci, and M. Tavani for useful discussions. We thank F. Vagnetti for the critical reading. A. Lapi thanks the SISSA/ISAS and INAF-OATS for warm hospitality.

\section{Appendix A: Analytic fit to the $\alpha$-profile}

To complement the analytical details extensively dealt with by Lapi \& Cavaliere (2009a) and to enable a straightforward comparison with the classic NFW and Einasto density runs, we provide here a handy analytic fit to the $\alpha$-profiles in terms of the deprojected Sérsic formula substantiated with parameters directly derived from the Jeans equation. We base it on the expression (see Prugniel \& Simien 1997)

$$
\frac{\rho(r)}{\rho\left(r_{-2}\right)}=\left(\frac{r}{r_{-2}}\right)^{-\tau} \exp \left\{-\frac{2-\tau}{\eta}\left[\left(\frac{r}{r_{-2}}\right)^{\eta}-1\right]\right\},
$$

where $\tau$ and $\eta$ are two fitting parameters. The standard Einasto profile is obtained by setting $\tau=0$. The values of $\tau$ and $\eta$ for different $\alpha$ of interest here are reported in Tables A.1 and A.2, both in the isotropic and the anisotropic cases. Note that $\tau \approx \gamma_{a}$ is required by the physical boundary condition satisfied at the center (see Sect. 2). The resulting fits to the density runs of the $\alpha$ profiles hold to better than $10 \%$ in the relevant range $10^{-2} r_{-2} \lesssim$ $r \lesssim 10 r_{-2}$.

The mass corresponding to the density distribution of Eq. (A1) writes as

$$
\frac{M(<r)}{M_{\infty}}=\Gamma\left[\frac{3-\tau}{\eta} ; \frac{2-\tau}{\eta}\left(\frac{r}{r_{-2}}\right)^{\eta}\right],
$$

where $\Gamma[a, x] \equiv \int_{0}^{x} \mathrm{~d} t t^{a-1} \mathrm{e}^{-t} / \int_{0}^{\infty} \mathrm{d} t t^{a-1} \mathrm{e}^{-t}$ is the (normalized) incomplete $\Gamma$-function.

\section{References}

Abadi, M. G., Navarro, F. N., Fardal, M., Babul, A., \& Steinmetz, M. 2009, MNRAS, submitted [arXiv:0902 . 2477]

Abdo, A. A., Ackermann, M., Ajello, M., et al. 2009, ApJS, 183, 46

Adriani, O., Barbarino, G. C., Bazilevskaya, G. A., et al. (PAMELA Collaboration) 2009, Nature, 458, 607

Arkani-Hamed, N., Finkbeiner, D. P., Slatyer, T., \& Weiner, N. 2009, Phys. Rev. D, 79, 015014

Ascasibar, Y., \& Gottlöber, S. 2008, ApJ, 386, 2022
Atwood, W. B., Abdo, A. A., Ackermann, M., et al. 2009, ApJ, 697, 1071 Austin, C. G., Williams, L. L. R., Barnes, E. I., Babul, A., \& Dalcanton, J. J. 2005, ApJ, 634, 756

Baer, H., \& Tata, X. 2009, in LHC physics, ed. A. Datta, B. Mukhopadhyaya, \& A. Raychaudhuri, in press [arXiv:0805.1905]

Baltz, E. A., et al. 2008, JCAP, 7, 13

Barbieri, R., Ferrara, S., \& Savoy, C. 1982, Phys. Lett. B, 119, 343

Bergström, L. 2009, in Dark Matter and Particle Physics, New J. Phys., 11, 105006

Bergström, L., Ullio, P., \& Buckley, J. H. 1998, Astropart. Phys., 9, 137 Bernabei, R., et al. 2008, Europhys. J. C, 56, 333

Bertone, G. 2009, Particle Dark Matter (Canbridge: Cambridge Univ. Press)

Bertone, G., Sigl, G., \& Silk, J. 2002, MNRAS, 337, 98

Bertone, G., Hooper, D., \& Silk, J. 2005, Phys. Rep., 405, 279

Bertone, G., Cirelli, M., Strumia, A., \& Taoso, M. 2009, JCAP, 3, 9

Binney J. 1978, MNRAS, 183, 779

Blumenthal, G. R., Faber, S. M., Flores, R., \& Primack, J. R. 1986, ApJ, 301, 27

Bullock, J. S., Kolatt, T. S., Sigad, Y., et al. 2001, MNRAS, 321, 559

Cavaliere, A., Lapi, A., \& Fusco-Femiano, R. 2009, ApJ, 698, 580

Cesarini, A., Fucito, F., Lionetto, A., Morselli, A., \& Ullio, P. 2004, Astrop. Phys., 21, 3, 267

Chamseddine, A., Arnowitt, R., \& Nath, P. 1982, Phys. Rev. Lett., 49, 970

Dehnen, W., \& McLaughlin, D. E. 2005, MNRAS, 363, 1057

Diemand, J., Kuhlen, M., \& Madau, P. 2007, ApJ, 667, 859

Diemand, J., Zemp, M., Moore, B., Stadel, J., \& Carollo, C. M. 2005, MNRAS, 364,665

El-Zant, A., Shlosman, I., \& Hoffman, Y. 2001, ApJ, 560, 636

Fornengo, N., Pieri, L., \& Scopel, S. 2004, Phys. Rev. D, 70, 3529

Gondolo, P., \& Silk, J. 1999, Phys. Rev. Lett. 83, 1719

Goodenough, L., \& Hooper, D. 2009, Fermilab-pub-09-494-A [arXiv: 0910.2998]

Graham, A. W., Merritt, D., Moore, B., Diemand, J., \& Terzić, B. 2006, AJ, 132, 2685

Hall, L., Lykken, J., \& Weinberg, S. 1983, Phys. Rev. D, 27, 2359

Hansen, S. H. 2004, MNRAS, 352, L41

Hansen, S. H., \& Moore, B. 2006, NewA, 11, 333

Hansen, B. V. 2007, B. S. Thesis, Univ. Copenhagen

Hoffman, Y., Romano-Díaz, E., Shlosman, I., \& Heller, C. 2007, ApJ, 671, 1108

Kormendy, J., Fisher, D. B., Cornell, M. E., \& Bender, R. 2009, ApJS, 182, 216

Lapi, A., \& Cavaliere, A. 2009a, ApJ, 692, 174

Lapi, A., \& Cavaliere, A. 2009b, ApJ, 695, L125

Lattanzi, M., \& Silk, J. 2009, Phys. Rev. D, 79, h3523

Lauer, T. R., Gebhardt, K., Faber, S. M., et al. 2007, ApJ, 664, 226

Li, Y., Mo, H. J., van den Bosch, F. C., \& Lin, W. P. 2007, MNRAS, 379, 689

Mo, H. J., Mao, S., \& White, S. D. M. 1998, MNRAS, 295, 319

Navarro, J. F., Ludlow, A., Springel, V., et al. 2010, MNRAS, 402, 21

Navarro, J. F., Frenk, C. S., \& White, S. D. M. 1997, ApJ, 490, 493

Ohta, N. 1983, Prog. Theor. Phys., 70, 542

Papucci, M., \& Strumia, A. 2009, CERN-PH-TH/2009-238 [arXiv: 0912.0742]

Peebles, P. J. E. 1993, Principles of Physical Cosmology (Princeton, NJ: Princeton Univ. Press)

Pieri, L., Pizzella, A., Corsini, E. M., Dalla Bontá, E., \& Bertola, F. 2009, A\&A, 496, 351

Prugniel, Ph., \& Simien, F. 1997, A\&A, 321, 111

Rasia, E., Tormen, G., \& Moscardini, L. 2004, MNRAS, 351, 237

Salvador-Solé, E., Manrique, A., González-Casado, G., \& Hansen, S. H. 2007, ApJ, 666, 181

Serpico, P. D., \& Hooper, D. 2009, in Dark Matter and Particle Physics, in press [arXiv: 0902.2539]

Dunkley, J., Komatsu, E., Nolta, M. R., et al. 2009, ApJ, 180, 306

Schmidt, K. B., Hansen, S. H., \& Macció, A. V. 2008, ApJ, 689, L33

Springel, V., White, S. D. M., Frenk, C. S., et al. 2008, Nature, 456, 73

Springel, V., Frenk, C. S., \& White, S. D. M. 2006, Nature, 440, 1137

Striani, E. 2009, B. S. Thesis, Univ. "Tor Vergata", Rome

Strigari, L. E. 2007, Phys. Rev. D, 75, 3526

Taylor, J. E., \& Navarro, J. F. 2001, ApJ, 563, 483

Tonini, C., Lapi, A., \& Salucci, P. 2006, ApJ, 649, 591

Vass, I., Valluri, M., Kravtsov, A., \& Kazantzidis, S. 2008, MNRAS, 395, 1225

Vitale, V., \& Morselli, A. 2009, Poster presented at the Fermi Symposium, 2-5 November, Washington DC, see

http: //fermi.gsfc.nasa.gov/science/symposium/2009/

Vitale, V., Morselli, A., et al. 2009, AIP Conf. Proc., 112, 164

Wechsler, R. H., Zentner, A. R., Bullock, J. S., Kravtsov, A. V., \& Allgood, B. 2006, ApJ, 652, 71

White, S. D. M. 1986, in Inner Space/Outer Space: the Interface between

Cosmology and Particle Physics (Chicago: Chicago Univ. Press), 228

Zhao, D. H., Mo, H. J., Jing, Y. P., \& Börner, G. 2003, MNRAS, 339, 12 